Published in final edited form as:

Neurobiol Dis. 2016 March ; 87: 124-133. doi:10.1016/j.nbd.2015.12.015.

\title{
PPAR $\boldsymbol{\gamma}$-induced Upregulation of CD36 Enhances Hematoma Resolution and Attenuates Long-term Neurological Deficits after Germinal Matrix Hemorrhage in Neonatal Rats
}

\author{
Jerry J. Flores ${ }^{1}$, Damon Klebe ${ }^{1}$, William B. Rolland ${ }^{1}$, Tim Lekic ${ }^{1}$, Paul R. Krafft ${ }^{1}$, and John \\ H. Zhang ${ }^{1,2}$ \\ 1'Department of Physiology \& Pharmacology, Loma Linda University School of Medicine, Loma \\ Linda, California USA \\ ${ }^{2}$ Departments of Anesthesiology and Neurosurgery, Loma Linda University School of Medicine, \\ Loma Linda, California, USA
}

\begin{abstract}
Germinal matrix hemorrhage remains the leading cause of morbidity and mortality in preterm infants in the United States with little progress made in its clinical management. Survivors are often afflicted with long-term neurological sequelae, including cerebral palsy, mental retardation, hydrocephalus, and psychiatric disorders. Blood clots disrupting normal cerebrospinal fluid circulation and absorption after germinal matrix hemorrhage are thought to be important contributors towards post-hemorrhagic hydrocephalus development. We evaluated if upregulating CD36 scavenger receptor expression in microglia and macrophages through PPAR $\gamma$ stimulation, which was effective in experimental adult cerebral hemorrhage models and is being evaluated clinically, will enhance hematoma resolution and ameliorate long-term brain sequelae using a neonatal rat germinal matrix hemorrhage model. PPAR $\gamma$ stimulation $\left(15 \mathrm{~d}-\mathrm{PGJ}_{2}\right)$ increased shortterm PPAR $\gamma$ and CD36 expression levels as well as enhanced hematoma resolution, which was reversed by a PPAR $\gamma$ antagonist (GW9662) and CD36 siRNA. PPAR $\gamma$ stimulation (15d-PGJ 2 ) also reduced long-term white matter loss and post-hemorrhagic ventricular dilation as well as improved neurofunctional outcomes, which were reversed by a PPAR $\gamma$ antagonist (GW9662). PPAR $\gamma$-induced upregulation of CD36 in macrophages and microglia is, therefore, critical for enhancing hematoma resolution and ameliorating long-term brain sequelae.
\end{abstract}

\section{Keywords}

PPAR $\gamma$; CD36; Germinal Matrix Hemorrhage; Post-hemorrhagic Ventricular Dilation; Hematoma Resolution

Corresponding author: John H. Zhang, MD PhD; Department of Physiology \& Pharmacology, Loma Linda University, Loma Linda, California, 92354, USA; Phone: 909-558-4723; Fax: 909-558-0119; johnzhang3910@ yahoo.com.

Publisher's Disclaimer: This is a PDF file of an unedited manuscript that has been accepted for publication. As a service to our customers we are providing this early version of the manuscript. The manuscript will undergo copyediting, typesetting, and review of the resulting proof before it is published in its final citable form. Please note that during the production process errors may be discovered which could affect the content, and all legal disclaimers that apply to the journal pertain. 


\section{Introduction}

The ganglionic eminence consists of neuronal and glial precursor cells located at the head of the caudate nucleus below the lateral ventricles of the developing fetus, and the highly vascularized region within the subependymal tissue is the germinal matrix. Cerebral blood flow fluctuation associated with hemodynamic and respiratory instability in preterm infants in conjunction with the inherent fragility of the germinal matrix often leads to germinal matrix hemorrhage, a very common and major neurological complication of prematurity. Germinal Matrix Hemorrhage (GMH) occurs when immature blood vessels rupture within the subependymal (or periventricular) germinal region of the ganglionic eminence in the immature brain (Ballabh, 2010). In the United States alone, GMH occurs in approximately 12,000 lives births per year, and the number of moderate-to-severe GMH cases has remained steady over the past two decades (Fanaroff et al., 2007; Jain et al., 2009; Osterman et al., 2015). Clinical studies indicate GMH afflicted infants often suffer from long-term neurological deficits, cerebral palsy, mental retardation, hydrocephalus, and psychiatric disorders (Ballabh, 2014; Kadri et al., 2006). Prenatal glucocorticoid treatment remains the best treatment for preventing GMH, yet minimal advancements have been made in GMH clinical management post-ictus (Roberts and Dalziel, 2006; Shankaran et al., 1995).

Hemodynamic and respiratory instability in preterm infants results in fluctuations of cerebral blood flow in the inherently frail germinal matrix vasculature, often resulting in spontaneous bleeding (Ballabh, 2014). The consequent hematoma applies mechanical pressure to glia and neurons, resulting in cytotoxicity and necrosis, as well as evokes an inflammatory response, leading to secretion of destructive proteases and oxidative species (Lekic et al., 2015). In adult cerebral hemorrhage, clinical studies indicate hematoma volume is the best prognostic indicator; larger hematoma volumes have worsened outcomes (Keep et al., 2005; Xi et al., 2006). Experimental adult cerebral hemorrhage studies proved more rapid hematoma resolution is necessary for quickly ameliorating inflammation and improving neurological recovery (Zhao et al., 2009; Zhao et al., 2007). Additionally, blood clots directly impair cerebrospinal fluid circulation and absorption after GMH, significantly contributing towards post-hemorrhagic hydrocephalus development (Aquilina et al., 2011; Cherian et al., 2004). Therefore, we hypothesize enhancing hematoma resolution will improve GMH outcomes.

Microglia are resident macrophages of the central nervous system and are critical drivers of the neuro-inflammatory response after GMH and other hemorrhagic brain injuries (Aronowski and Zhao, 2011; Tang et al., 2015). Activated microglia recruit hematogenous phagocytes to the injured site, which engulf the hematoma as well as damaged or dead tissue (Aronowski and Hall, 2005; Cox et al., 1995). The role microglia play in hemorrhagic brain injury pathogenesis is different in neonates than adults (Woo et al., 2012). Unlike the adult brain where microglia cells and macrophages contribute to brain injury after stroke through the production of inflammatory cytokines (Vexler and Yenari, 2009), neonatal brains demonstrate the opposite as the depletion of these cells enhances injury by removing endogenous protective mechanisms (Faustino et al., 2011).

Scavenger receptor CD36, a trans-membrane glycoprotein, is involved in several biological functions, such as foam cell formation, immune cell chemotaxis, and phagocytosis of 
apoptotic cells (Woo et al., 2012). CD36 receptor is reportedly located on the cell surface of several cell types, including monocytes, endothelial cells, and microglia. CD36 plays an important role in phagocytosis, and upregulating its expression beneficially enhances hematoma resolution (Zhao et al., 2007). Transfection of non-phagocytic cells with a CD36expressing gene converted those cells into phagocytes (Ren et al., 1995). CD36 genetic deletion worsened injury after acute focal stroke in neonatal mice, partially by decreasing removal of apoptotic cells (Woo et al., 2012).

Peroxisome proliferator-activated receptor gamma (PPAR $\gamma$ ), a member of the nuclear hormone receptor superfamily, plays a major role in the upregulating CD36 expression (Zhao et al., 2009; Zhao et al., 2007). PPAR $\gamma$ stimulation exerts anti-inflammatory effects in several central nervous system injuries and disorders (Landreth et al., 2008; Pereira et al., 2005). Many studies demonstrated PPAR $\gamma$ is neuroprotective in various experimental stroke models (Pereira et al., 2005; Shao and Liu, 2015; Woo et al., 2012; Zhao et al., 2007). In adult intracerebral hemorrhage (ICH) experimental models, PPAR $\gamma$ activation was directly associated with upregulation of CD36 expression, leading to enhanced phagocytosismediated clearance of the hematoma as well as dead or damaged cells by microglia and macrophages. PPAR $\gamma$ stimulation reduced expression of pro-inflammatory mediators, ameliorated secondary brain injury, and improved functional recovery after ICH (Zhao et al., 2007). Currently, PPAR $\gamma$ stimulation for enhancing hematoma resolution and ameliorating secondary brain injury is being clinically tested in ICH patients (Gonzales et al., 2013). Yet, no clinical trials are evaluating PPAR $\gamma$ stimulation in GMH patients.

In this study, we assess if PPAR $\gamma$ stimulation enhances CD36-mediated hematoma resolution in a neonatal rat germinal matrix hemorrhage model. We hypothesize PPAR $\gamma$ stimulation, using $15 \mathrm{~d}-\mathrm{PGJ}_{2}$, will augment microglia/macrophage phagocytosis of blood clots, reducing post-hemorrhagic hydrocephalus, inflammation, behavioral dysfunction, and neuronal loss, which will be reversed by CD36 knockdown or PPAR $\gamma$ antagonist administration.

\section{Materials and methods}

\section{Animals and surgeries}

All experimental procedures were conducted in accordance with the National Institutes of Health guidelines for the treatment of animals, and were approved by the Institutional Animal Care and Use Committee of Loma Linda University. Two hundred and sixty P7 Sprague-Dawley neonatal pups (Harlan, Indianapolis, IN) weighing 12-15 g (brain development is comparable to 30-32 week gestation humans) were used in this study. Germinal matrix hemorrhage was achieved by stereotactic-guided injection of bacterial collagenase, as previously described (Lekic et al., 2012). Pups were anesthetized with 3\% isoflurane (delivered through medical grade oxygen and mixed air) while being stabilized onto a stereotaxic frame. Isopropyl alcohol followed by betadine was applied to the incision site. Incision was made on the longitudinal plane to expose the skull and reveal bregma. The stereotactic coordinates from bregma were as follows: $1.6 \mathrm{~mm}$ (rostral), $1.6 \mathrm{~mm}$ (right lateral), and $2.8 \mathrm{~mm}$ (depth) from the dura. A burr hole $(1 \mathrm{~mm})$ was drilled, into which a 27 gauge needle was inserted at a rate of $1 \mathrm{~mm} / \mathrm{min}$. 0.3 units of clostridial collagenase VII-S 
(Sigma Aldrich, MO) in $1 \mu \mathrm{L}$ was infused over a period of 3 minutes with a Hamilton syringe guided by a microinfusion pump (Harvard Apparatus, Holliston, MA). After completing infusion, the needle is left into position for an additional 10 minutes after injection to prevent "back-leakage" and then removed at a rate of $1 \mathrm{~mm} / \mathrm{min}$. Once the Hamilton is removed, the burr hole is sealed with bone wax and the incision site sutured. Animals are then given buprenorphine and allowed to recover on a $37^{\circ} \mathrm{C}$ heated blanket. When fully recovered, pups are then placed back with the mother. Surgery time per animal is approximately 30 minutes. Sham animals were subject to needle insertion without collagenase infusion. The same procedures were performed for intraventricular injection of siRNA, except the stereotactic coordinates from bregma were as follows: $1.0 \mathrm{~mm}$ (rostral), $1.0 \mathrm{~mm}$ (left lateral), and $1.8 \mathrm{~mm}$ (depth) of the dura.

\section{Animal Treatments and experimental groups}

P7 rat pups were randomly divided into the following groups: sham-operated $(n=38)$, Vehicle (n=38), GMH + 15d-PGJ 2 (n=38), GMH + 15d-PGJ $2+$ GW9662 (n=38), GMH + CD36 siRNA ( $\mathrm{n}=12), \mathrm{GMH}+15 \mathrm{~d}-\mathrm{PGJ}_{2}+\mathrm{CD} 36(\mathrm{n}=12), \mathrm{GMH}+$ scrambled siRNA $(\mathrm{n}=12)$. The PPAR $\gamma$ agonist (15d-PGJ $2,0.1 \mathrm{mg} / \mathrm{kg}$; Sigma Aldrich), antagonist (GW9662, 4mg/kg; Sigma Aldrich) + agonist, and saline (for sham and vehicle groups) were administered intraperitoneally to experimental animals at 1 hour post-GMH and then once daily for 7 days. CD36 siRNA (1.2 ng, Cell Signaling \& Santa Cruz), and scrambled siRNA (1.2 ng, Santa Cruz) were administered via intraventricular injection to experimental animals 24 hours prior to GMH induction. Buprenorphine $(0.01 \mathrm{mg} / \mathrm{kg})$ was administered subcutaneously to all groups after completing surgery.

\section{Animal Perfusion and Tissue Extraction}

Animals were euthanized using isoflurane ( $25 \%$ ) followed by trans-cardiac perfusion with ice-cold phosphate buffered saline (PBS) for hemoglobin assay and Western blot samples or with ice-cold PBS followed by $10 \%$ formalin for histology samples. Forebrains for hemoglobin assay and Western blot were snap-frozen with liquid-nitrogen, then stored in $-80^{\circ} \mathrm{C}$ freezer before protein extractions or spectrophotometric quantification. Histological brain samples were post-fixed in 10\% Fomaldehyde for at least 3 days and then $30 \%$ sucrose for at least 3 days, all stored in $4^{\circ} \mathrm{C}$ fridge. Forebrains were next embedded in Optimal Cutting Temperature compound and stored in $-20^{\circ} \mathrm{C}$ freezer.

\section{Hemoglobin Assay}

Spectrophotometric measurements were used to assess hemorrhagic volume using wellestablished protocols (Choudhri et al., 1997; Lekic et al., 2011; Tang et al., 2004). Frozen extracted forebrains were placed into individual glass tubes containing $3 \mathrm{~mL}$ of PBS. The tissue was homogenized for 60 seconds (Tissue Miser Homogenizer; Fisher Scientific, Pittsburgh, PA) followed by ultrasonication for 1 minute to lyse erythrocyte membranes. The products were then centrifuged for 30 minutes and the supernatant was separated from the pellets. A 4:1 ratio of Drabkin's reagent (Sigma-Aldrich) and supernatant were combined, which were left to react for 15 minutes. Absorbance, using a spectrophotometer (540 nm; Genesis 10uv; Thermo Fisher Scientific, Waltham, MA), was calculated into a 
hemorrhagic volume $(\mu \mathrm{L})$ on the basis of a standard curve as routinely performed (Lekic et al., 2011).

\section{Intracranial Pressure (ICP) Measurements}

At 28 days post-ictus, animals were anesthetized and mounted onto a stereotaxic frame, where the head was inclined downward at a 30 degree angle. A midline skin incision was made to expose the atlanto-occipital membrane. The cisterna magna was punctured with a 26G Hamilton needle, which was connected to a pressure transducer of a Digi-Med LPA 400-low pressure Anayzer (Micro-Med-Louisville, Kentucky, USA) as described (Lackner et al., 2013)

\section{Western Blotting}

Protein concentrations for immunoblot (Lekic et al., 2011) were determined by DC protein assay (Bio-Rad, Hercules, CA). $30 \mu \mathrm{g}$ protein per sample were loaded into wells of 4-20\% gels, ran for 30 minutes at $50 \mathrm{~V}$ then 90 minutes at $125 \mathrm{~V}$, then transferred onto nitrocellulose membranes at $0.3 \mathrm{~A}$ for 120 minutes (Bio-Rad). Membranes were incubated for 2 hours in $5 \%$ non-fat milk in Tris-buffered saline containing $0.1 \%$ Tween 20 . Then the following primary antibodies were incubated overnight: anti-PPARy (1:1000; Santa Cruz, Dallas, Texas), CD36 (1:500, Santa Cruz, Dallas, Texas), and mannose receptor (CD206) (1:500, ABcam, Cambridge, Massachusetts). Secondary antibodies (1:2000; Santa Cruz Biotechnology, Santa Cruz, CA) were then applied to the membranes and incubated for 2 hours and then processed with the ECL plus Kit (GE Healthcare and Life Science, Piscataway, NJ). $\beta$-actin was used as an internal control against the anti-body (1:1000; Santa Cruz Biotechnology, Santa Cruz, CA). ImageJ software (4.0, Media Cybernetics, Silver Spring, MD) was used to analyze the relative density of the resultant protein immunoblot images as described (Tang et al., 2004).

\section{Histological Volumetric Analysis}

$10 \mu \mathrm{m}$ thick coronal brain sections were cut every $600 \mu \mathrm{m}$ using a cryostat (Leica Microsystems LM3050S) and were placed onto poly-L-lysine-coated slides. Brain slices were Nissl stained morphometrically analyzed using computer-assisted (ImageJ 4.0, Media Cybernetics, Silver Spring, MD) hand delineation of the ventricle system (lateral, third, cerebral aqueduct, and fourth), hemisphere (cortex, subcortex), caudate, thalamus, hippocampus, and corpus callosum (white matter) (Lekic et al., 2011). These structures were delineated using optical dissector principles from prior stereological studies (Avendano et al., 2005; Bermejo et al., 2003; Ekinci et al., 2008; Klebe et al., 2014; Oorschot, 1996; Reisert et al., 1984; Tang et al., 2001). Volumes were calculated using the following equation: [(Average [(Area of coronal section) $\times$ Interval $\times$ Number of sections) (MacLellan et al., 2008).

\section{Immunohistochemistry}

$10 \mu \mathrm{m}$ thick slices were first stained with OX-42 (1:1000, ABcam) and mannose receptor (1:1000, ABcam) overnight at $4{ }^{\circ} \mathrm{C}$, followed by incubation with appropriate fluorescence conjugated secondary antibodies (Jackson Immunoresearch, West Grove, PA). The peri- 
hemorrhagic area was imaged by a Fluorescent Olympus-BX51 microscope and analyzed using MagnaFire SP 2.1B software (Olympus, Melville, NY). At least six sections per animal group over a microscopic field of $20 \times$ (for microglia) were averaged and expressed as cells/field, as described (Wang and Dore, 2007)

\section{Neurobehavioral Analysis}

Neurobehavioral function was evaluated in a blinded manner using a battery of tests, described below, to detect sensorimotor and cognitive deficits 28 days after GMH as previously described (Hartman et al., 2009; Klebe et al., 2014): Foot Fault Test: Rats were placed on a wire grid $(20 \times 40 \mathrm{~cm})$ kept above the floor level and allowed to walk on the grid for 2 minutes. Number of foot faults will be recorded when a complete paw falls through the openings in the grid. Rotarod Test: Rats were placed on a rotarod (Columbus Instruments, Columbus, $\mathrm{OH}$ ), which consists of a rotating horizontal cylinder $(7 \mathrm{~cm}$ diameter $)$ divided into 9.5-cm-wide lanes. Rats walked forward when the cylinder is rotating to avoid falling down. Rats were tested at a starting 5 RPM or 10 RPM with acceleration at 2 RPM per 5 seconds. A photobeam circuit detected the latency to fall off the cylinder. Water Maze

Test: Rats were released in a metal pool $(110 \mathrm{~cm}$ diameter) filled with water and containing spatial cues on the walls, and they were allowed to swim to find a submerged platform (11 $\mathrm{cm}$ diameter). Each animal performed 10 trials per day for 4 days, 5 blocks of 2 consecutive trials, with a 10-min interval between successive blocks. Over the next 3 days, the platform was submerged $1 \mathrm{~cm}$ below the water and surface and the rats had to find and remember the platform location. Additionally, at the end of each day, the platform was removed and animals were allowed to swim to find the platform quadrant. An overhead camera with a computerized tracking system (Noldus Ethovision; Noldus, Tacoma, WA) recorded the swim path and measured the swim distance, swim speed, and time spent in probe quadrant.

\section{Statistical Analysis}

A power analysis using a type I error rate of 0.05 and a power of 0.8 on a 2-sided test was used to estimate sample size. Data are expressed in Mean \pm Standard Deviation. One-way ANOVA on ranks using the Student-Newman-Keuls post-hoc test was used to analyze behavioral, histological, western blots, and immunohistochemistry. A P-value $<0.05$ was considered statistically significant.

\section{Results}

\section{PPAR $\gamma$ Stimulation Ameliorated Long-term Neurological Deficits}

The vehicle group performed significantly worse compared to sham in the Morris Water Maze evaluation, yet PPAR $\gamma$ stimulation improved spatial learning and memory compared to vehicle and was not significantly different from sham. Treatment effect was reversed by the PPAR $\gamma$ antagonist $(* \mathrm{P}<0.05$ versus Sham; $\# \mathrm{P}<0.05$ versus Vehicle; $\dagger \mathrm{P}<0.05$ versus GW9662 + 15d-PGJ 2 ; Figure 1.A-B). Treatment also significantly improved sensorimotor function in the foot fault test compared to other GMH groups $(* \mathrm{P}<0.05$ versus Sham; $\# \mathrm{P}<0.05$ versus Vehicle; $\dagger \mathrm{P}<0.05$ versus GW9662 $+15 \mathrm{~d}-\mathrm{PGJ}_{2} ;$ Figure 1.C). Yet no significant difference was achieved in the treated group compared to vehicle and antagonist 
groups in the rotarod sensorimotor evaluation $(* \mathrm{P}<0.05$ versus Sham; \#P<0.05 versus Vehicle; $† \mathrm{P}<0.05$ versus GW9662 + 15d-PGJ 2 ; Figure 1.D).

\section{PPAR $\gamma$ Stimulation Improved Long-term Brain Morphology}

Since increased intracranial pressure is associated with hydrocephalus, ICP was measured in rats at 4 weeks post-ictus. ICP was significantly decreased in treated groups when compared to vehicle and PPAR $\gamma$ antagonist groups ( ${ }^{*} \mathrm{P}<0.05$ versus Sham; $\# \mathrm{P}<0.05$ versus Vehicle; $\dagger \mathrm{P}<0.05$ versus GW9662 $+15 \mathrm{~d}-\mathrm{PGJ}_{2}$; Figure 2.B). Calculated cortical thickness is presented as a ratio to the mean of sham and was significantly deceased in the vehicle and PPAR $\gamma$ antagonist group, but the PPAR $\gamma$ stimulated group had significantly less cortical loss ( $* \mathrm{P}<0.05$ versus Sham; \#P $<0.05$ versus Vehicle; $\dagger \mathrm{P}<0.05$ versus $\mathrm{GW} 9662+15 \mathrm{~d}-\mathrm{PGJ}{ }_{2}$; Figure 3.A). Ventricular volume was significantly increased in vehicle and PPAR $\gamma$ antagonist groups, but $15 \mathrm{~d}-\mathrm{PGJ}_{2}$ treatment reduced post-hemorrhagic ventricular dilation ( $* \mathrm{P}<0.05$ versus Sham; $\# \mathrm{P}<0.05$ versus Vehicle; $\uparrow \mathrm{P}<0.05$ versus $\mathrm{GW} 9662+15 \mathrm{~d}-\mathrm{PGJ}_{2}$; Figure 3.B). White matter loss is presented as a percentage of white matter present to mean of sham. White matter loss was reduced by PPAR $\gamma$ stimulation, but vehicle and PPAR $\gamma$ antagonist groups had significant white matter loss $(* \mathrm{P}<0.05$ versus $S$ Sham; \#P $<0.05$ versus Vehicle; $† \mathrm{P}<0.05$ versus GW9662 $+15 \mathrm{~d}-\mathrm{PGJ}_{2}$; Figure 3.C). Basal ganglia loss is presented as a percentage of basal ganglia present to mean of sham. The $15 \mathrm{~d}-\mathrm{PGJ}_{2}$ treated group had significantly decreased basal ganglia loss compared to the vehicle group. Surprisingly, basal ganglia loss in the GW9662 group was not significantly different from PPAR $\gamma$ agonist or vehicle groups $(* \mathrm{P}<0.05$ versus Sham; $\# \mathrm{P}<0.05$ versus Vehicle; $\dagger \mathrm{P}<0.05$ versus GW9662+ $15 \mathrm{~d}-\mathrm{PGJ}_{2}$; Figure 3.D).

\section{PPAR $\gamma$ Stimulation Enhanced Hematoma Resolution, Increased Activated Microglia, induce M2 Polarization}

A hemoglobin assay time-course was conducted at 24 hours, 72 hours, and 7 days to determine PPAR $\gamma$ 's role in hematoma resolution. At 24 hours, all groups had significantly greater hemoglobin content in the brain compared to sham $(* \mathrm{P}<0.05$ versus Sham; Figure 4.A). At 72 hours, all groups had significantly greater hemoglobin content in the brain compared to sham, but $15 \mathrm{~d}-\mathrm{PGJ}_{2}$ treatment had significantly less hemoglobin content compared to vehicle, which was reversed by GW9662 treatment $(* \mathrm{P}<0.05$ versus Sham; $\# \mathrm{P}<0.05$ versus Vehicle; $\dagger \mathrm{P}<0.05$ versus GW9662 + 15d-PGJ 2 ; Figure 4.B). At 7 days, only the vehicle and PPAR $\gamma$ antagonist groups had significantly greater hemoglobin content compared to sham, but the PPAR $\gamma$ agonist group had significantly less hemoglobin content compared to vehicle and $\mathrm{PPAR} \gamma$ antagonist groups $\left({ }^{*} \mathrm{P}<0.05\right.$ versus Sham; \#P $<0.05$ versus Vehicle; $† P<0.05$ versus GW9662 +15 d-PGJ 2 ; Figure 4.C). Since significantly greater hematoma resolution was observed in the PPAR $\gamma$ stimulated group at 72 hours, representative photographs of immuno-stained activated microglia/macrophages (OX-42) in the peri-hematoma region are presented at this time point (Figure 5.A). The $15 \mathrm{~d}-\mathrm{PGJ}_{2}$ treated group had relatively increased number of microglia/macrophage compared to sham, vehicle, and PPAR $\gamma$ antagonist group $((* \mathrm{P}<0.05$ versus Sham; $\# \mathrm{P}<0.05$ versus Vehicle; $\dagger \mathrm{P}<0.05$ versus GW9662 $+15 \mathrm{~d}-\mathrm{PGJ}_{2}$; Figure 5.B). Additionally representative photographs of the co-localization of activated microglia/macrophages (OX-42) and mannose receptor (CD206) in the peri-hematoma region are presented at 72 hours, showing an increase in 
OX-42/mannose receptor expression in $15 \mathrm{~d}-\mathrm{PGJ}_{2}$ treated group in comparison to all other groups (Figure 5.A).

\section{PPAR $\gamma$ Stimulation Increased CD36 and PPAR $\gamma$ Expression at 72 Hours}

A time-course study was conducted to determine endogenous PPAR $\gamma$ and CD36 expression levels in GMH at 0, 3, 6, 12 hours and 1, 3, 5, and 7 days after GMH. Endogenous PPAR $\gamma$ expression was significantly increased at $3,6,12$ hours and 1 day compared to 0 hour $(* \mathrm{P}<0.05$ versus 0 hours; Figure 6.A). Correspondingly, endogenous CD36 expression was significantly increased at $3,6,12$ hours, and 1 day compared to 0 hour $(* \mathrm{P}<0.05$ versus 0 hours; Figure 6.B), and tended to remain elevated by 7 days. PPAR $\gamma$ and CD36 expression levels were determined at 72 hours for all experimental groups. PPAR $\gamma$ expression was significantly increased in the $15 \mathrm{~d}-\mathrm{PGJ}_{2}$ group compared to all other groups $(* \mathrm{P}<0.05$ versus Sham; \#P<0.05 versus Vehicle; $† \mathrm{P}<0.05$ versus GW9662 $+15 \mathrm{~d}-\mathrm{PGJ}_{2}$; Figure 6.C). Much like PPAR $\gamma, \mathrm{CD} 36$ expression was significantly increased in the $15 \mathrm{~d}-\mathrm{PGJ} \mathrm{J}_{2}$ group compared to all other groups $(* \mathrm{P}<0.05$ versus Sham; $\# \mathrm{P}<0.05$ versus Vehicle; $\uparrow \mathrm{P}<0.05$ versus GW9662 + 15d-PGJ 2 ; Figure 6.D).

\section{CD36 Knockdown Reversed PPAR $\gamma$ Agonist-enhanced Hematoma Resolution and M2 expression at 72 hours}

At 72 hours, CD36 knockdown reversed PPAR $\gamma$ agonist-enhanced hematoma resolution, which was not reversed in the scrambled siRNA group $(* \mathrm{P}<0.05$ versus Sham, $\# \mathrm{P}<0.05$ versus Vehicle, $\% \mathrm{P}<0.05$ versus $\mathrm{CD}-36$ siRNA + 15d-PGJ 2 , @ $\mathrm{P}<0.05$ versus scrambled siRNA+15d-PGJ 2 , Figure 4.D). Additionally, CD36 knockdown reversed PPAR $\gamma$ mannose receptor expression, which was not reversed in the scrambled siRNA group $(* \mathrm{P}<0.05$ versus Sham, \#P<0.05 versus Vehicle, $\% \mathrm{P}<0.05$ versus CD-36 siRNA + 15d-PGJ 2 , @ $\mathrm{P}<0.05$ versus scrambled siRNA+15d-PGJ 2 , Figure 5.C).

\section{Discussion}

Neonatal brain hemorrhage is a common affliction of premature infants. The resultant hematoma is thought to play a major role in causing post-hemorrhagic hydrocephalus development because blood clots disrupt cerebrospinal fluid circulation and absorption in the ventricles (Crews et al., 2004). Rapid hematoma resolution was neuroprotective in adult hemorrhagic stroke models (Zhao et al., 2015; Zhao et al., 2007). In particular, PPAR $\gamma$ stimulation upregulates CD36 expression in microglia/macrophages, leading to increased phagocytosis of blood products and more rapid hematoma resolution after adult intracerebral hemorrhage (Zhao et al., 2007). In this study, we examined the effects of stimulating PPAR $\gamma$ with $15 \mathrm{~d}-\mathrm{PGJ}_{2}$ on hematoma resolution as well as long-term brain morphological and neurofunctional outcomes after collagenase-induced GMH in neonatal rats. In addition, we determined if PPAR $\gamma$ and CD36 inhibition reversed observed therapeutic effects from PPAR $\gamma$ stimulation by $15 \mathrm{~d}-\mathrm{PDJ}_{2}$. This study is the first to assess this treatment approach in neonates for enhancing hematoma resolution as well as the first to evaluate its therapeutic potential for ameliorating long-term white matter loss, post-hemorrhagic hydrocephalus development, and neurological deficits. 
We evaluated the efficacy of $15 \mathrm{~d}-\mathrm{PGJ}_{2}$ treatment, a PPAR $\gamma$ agonist, administered 1 hour post-ictus as a potential therapeutic modality for GMH-induced brain injury. Although treatment did not significantly increase hematoma resolution at 24 hours, it did significantly enhance hematoma resolution at 72 hours and 7 days after GMH (Figure 4.A-C). PPAR $\gamma$ stimulation demonstrated more rapid hematoma clearance after GMH in neonates, starting at 72 hours, than after ICH in adults, which took 7 days (Zhao et al., 2007). 15d-PGJ 2 coadmiration with GW9662, a PPAR $\gamma$ antagonist, reversed $15 \mathrm{~d}_{-} \mathrm{PGJ}_{2}$ treatment effects on enhanced hematoma resolution at 72 hours and 7 days after GMH. To determine if endogenous expression of PPAR $\gamma$ and CD36 changes after GMH, we performed a Western blot time course using 0,3, 6, 12 hour, 1, 3, 5, and 7 day endpoints following GMH induction. Endogenous PPAR $\gamma$ expression increased at 3, 6, 12 hour, and 1 day after GMH induction, then returned to baseline by 3 days (Figure 6.A). Endogenous CD36 expression significantly increased at 3,6,12 hours, and 1 day after GMH induction, and tended to remain elevated through 7 days (Figure 6.B). Because endogenous PPAR $\gamma$ and CD36 expression returned near baseline at 72 hours and because $15 \mathrm{~d}-\mathrm{PGJ}_{2}$ treatment enhanced hematoma resolution at 72 hours, we determined treatment effects on PPAR $\gamma$ and CD36 expression levels by Western blot at 72 hours. As expected, $15 \mathrm{~d}-\mathrm{PGJ}_{2}$ treatment significantly increased PPAR $\gamma$ expression compared to sham and vehicle, and $15 \mathrm{~d}-\mathrm{PGJ}_{2}$ coadministration with GW9662 reversed this effect (Figure 6.C). Similarly, $15 \mathrm{~d}-\mathrm{PGJ}_{2}$ treatment significantly increased CD36 expression compared to sham and vehicle, and 15d$\mathrm{PGJ}_{2}$ co-administration with GW9662 reversed this effect (Figure 6.D).

CD36 receptor is an important scavenger receptor located on several cell types, including monocytes, endothelial cells, and microglia/macrophages. CD36 plays an important role in microglia/macrophage phagocytosis, and upregulating its expression beneficially enhances hematoma resolution (Zhao et al., 2007). More activated microglia were observed in the peri-hematoma region of treated GMH animals than all other groups, providing evidence of PPAR $\gamma$ 's treatment effects are dependent upon these critical immune cells (Figure 5.A-B). All GMH groups seemed to have increased DAPI stained cells post-ictus compared to sham. The germinal matrix has many growing and dividing neuronal and glial precursor cells. Prior GMH studies have documented increased proliferative cytokines and pathways, such as TGF- $\beta$ and mTOR, after injury, and we speculate proliferative signaling triggers more profound gliosis after neonatal brain hemorrhage (Lekic et al., 2015). While brain cell death is definitely occurring, we speculate proliferative signaling in conjunction with increased leukocyte infiltration contributes towards these observed results in neonatal brains. Although activated microglia/macrophages contribute to brain injury in adults, some evidence suggests microglia/macrophages have important defense mechanisms against injury in neonates, which could be explained by the vital role microglia play in neonatal brain development (Faustino et al., 2011; Harry and Kraft, 2012). Furthermore, activated microglia/macrophages have two differentiated states, a pro-inflammatory classically activated state (M1), and an immune dampening and tissue regenerative alternatively activated state (M2) (Klebe et al., 2015). CD36 stimulation can contribute towards microglia/macrophage activation as well as M2 polarization (Chavez-Sanchez et al., 2014; Kouadir et al., 2012; Rios et al., 2013). PPAR $\gamma$ stimulation also polarizes microglia/ macrophages towards the M2 state (Penas et al., 2015; Pisanu et al., 2014; Yoon et al., 
2008). To corroborate these findings in GMH, immunohistochemical co-localization of activated microglia and mannose receptor (CD206), an M2 marker, demonstrated increased co-expression in $15 \mathrm{~d}-\mathrm{PGJ}_{2}$ treated GMH animals compared to other groups, providing more evidence PPAR $\gamma$ induces M2 polarization (Figure 5.A). Similarly, CD206 Western blots demonstrated increased CD206 expression levels in treated GMH animals compared to sham, GMH animals with CD36 siRNA alone, and GMH animals with CD36 siRNA and $15 \mathrm{~d}-\mathrm{PGJ}_{2}$ treatment. Interestingly, vehicle treated GMH animals showed a small tendency towards having increased CD206 expression levels, which also did not achieve a statistically significant difference compared to $15 \mathrm{~d}-\mathrm{PGJ}_{2}$ treated GMH animals. After hemorrhage, M2 microglia/macrophages are expected to increase over time as the peri-hematoma milieu transitions into an immune dampened $t$ issue repair phase in which M2 microglia/ macrophages play a pivotal role (Klebe et al., 2015). CD36 knockdown eliminated the tendency observed in the vehicle group, achieving a significantly reduced expression compared to $15 \mathrm{~d}-\mathrm{PGJ} \mathrm{J}_{2}$ treatment. CD36 knockdown also reversed $15 \mathrm{~d}-\mathrm{PGJ}_{2}$ induced upregulation of CD206 expression (Figure 5.C). CD36, thus, is important for M2 polarization, particularly after PPAR $\gamma$ stimulation. To further confirm microglial/ macrophage CD36 plays a pivotal role in PPAR $\gamma$-induced blood clot clearance, siRNA was used to knockdown CD36 expression. CD36 knockdown reversed 15d-PGJ 2 treatment effects on enhanced hematoma resolution at 72 hours, which was not reversed by scrambled siRNA (Figure 4.D).

In our long-term evaluations, vehicle treated GMH animals had significant cortical, white matter, and basal ganglia loss as well as post-hemorrhagic ventricular dilation, but $15 \mathrm{~d}-\mathrm{PGJ}_{2}$ treatment ameliorated these brain morphological maladies, which were reversed by PPAR $\gamma$ antagonist, GW9662, co-administration (Figure 3.A-D). Surprisingly, GW9662 coadministration did not completely reverse $15 \mathrm{~d}-\mathrm{PGJ}_{2}$ 's effects on reducing basal ganglia loss, although a tendency was observed. Our ICP measurements agreed with the brain morphological assessment, where ICP levels were significantly lower in treated groups when compared to vehicle and PPAR $\gamma$ antagonist group (Figure 2.B). Additionally, vehicle treated GMH animals performed poorly in the Morris Water Maze, Foot Fault, and Rotarod tests, but $15 \mathrm{~d}-\mathrm{PGJ}_{2}$ treatment significantly improved spatial memory and motor function, which were reversed by GW9662 co-administration (Figure 1.A-D). The foot fault test evaluates locomotor function, the rotarod test evaluates sensorimotor coordination and balance, and Morris Water Maze evaluates spatial learning and memory (Schaar et al., 2010). Although 15d-PGJ2 reduced the number of foot faults, it did not significantly improve performance in the rotarod test. GMH and consequent post-hemorrhagic ventricular dilation may cause cerebellar injury that affects motor coordination (Brouwer et al., 2015; Fumagalli et al., 2015; Volpe, 2009). More thorough investigations are needed to elucidate the pathophysiology between GMH and cerebellar injury. Although motor coordination is an important factor in both the rotarod and foot fault tests, the element of balancing on an accelerating cylinder platform leads us to speculate cerebellar injury may more profoundly affect performances in the rotarod evaluations. 15d-PGJ2 treatment may not be effective enough to ameliorate potential cerebellar injury from GMH and consequent posthemorrhagic hydrocephalus, and, if this is the case, modulating the immune response after GMH may not be sufficient to promote complete functional recovery. Additionally, the 
rotarod test may not be sensitive enough for detecting improved sensorimotor outcomes in treatment groups, which is why we performed multiple neurofunctional evaluations.

Nonetheless, these results provide sufficient evidence that enhanced hematoma resolution corresponds with improved long-term brain morphological and neurocognitive outcomes after GMH. Most importantly, more rapid blood clot clearance resulted in significantly decreased post-hemorrhagic ventricular dilation, providing evidence that blood products play an important role in post-hemorrhagic hydrocephalus development.

The CD36 scavenger receptor is important in microglia/macrophage-mediated phagocytosis of cellular debris and blood products. Our results suggest PPAR $\gamma$ stimulation by $15 d-P G J_{2}$ increases microglia/macrophage phagocytic function by upregulating CD36 scavenger receptor expression in our experimental GMH model, leading to enhanced hematoma resolution. We are first to report the positive long-term effects on brain morphological and neurofunctional outcomes from more efficient hematoma resolution after GMH. Blood products disrupt cerebrospinal circulation and absorption in the cerebroventricular system, often resulting in post-hemorrhagic hydrocephalus. Herein, we provide evidence that more rapid blood clot clearance reduces long-term post-hemorrhagic ventricular dilation after GMH. Removing the hematoma without damaging surrounding tissues is ideal and clinically relevant. PPAR $\gamma$ stimulation could be a promising therapeutic approach for GMH patients, especially since PPAR $\gamma$ stimulation by Pioglitazone is already being evaluated in clinical trials for adult cerebral hemorrhage.

\section{References}

Aquilina K, et al. Neonatal rat model of intraventricular haemorrhage and posthaemorrhagic ventricular dilatation with long-term survival into adulthood. Neuropathol Appl Neurobiol. 2011; 37:156-165. [PubMed: 20819170]

Aronowski J, Hall CE. New horizons for primary intracerebral hemorrhage treatment: experience from preclinical studies. Neurol Res. 2005; 27:268-279. [PubMed: 15845210]

Aronowski J, Zhao X. Molecular pathophysiology of cerebral hemorrhage: secondary brain injury. Stroke. 2011; 42:1781-1786. [PubMed: 21527759]

Avendano $\mathrm{C}$, et al. Neuron numbers in the sensory trigeminal nuclei of the rat: A GABA- and glycineimmunocytochemical and stereological analysis. J Comp Neurol. 2005; 493:538-553. [PubMed: 16304625]

Ballabh P. Intraventricular hemorrhage in premature infants: mechanism of disease. Pediatr Res. 2010; 67:1-8. [PubMed: 19816235]

Ballabh P. Pathogenesis and prevention of intraventricular hemorrhage. Clin Perinatol. 2014; 41:4767. [PubMed: 24524446]

Bermejo PE, et al. Quantitative stereological evaluation of the gracile and cuneate nuclei and their projection neurons in the rat. J Comp Neurol. 2003; 463:419-433. [PubMed: 12836177]

Brouwer MJ, et al. Effects of Posthemorrhagic Ventricular Dilatation in the Preterm Infant on Brain Volumes and White Matter Diffusion Variables at Term-Equivalent Age. J Pediatr. 2015

Chavez-Sanchez L, et al. The role of TLR2, TLR4 and CD36 in macrophage activation and foam cell formation in response to oxLDL in humans. Hum Immunol. 2014; 75:322-329. [PubMed: 24486576]

Cherian S, et al. The pathogenesis of neonatal post-hemorrhagic hydrocephalus. Brain Pathol. 2004; 14:305-311. [PubMed: 15446586]

Choudhri TF, et al. Use of a spectrophotometric hemoglobin assay to objectively quantify intracerebral hemorrhage in mice. Stroke. 1997; 28:2296-2302. [PubMed: 9368579] 
Cox G, et al. Macrophage engulfment of apoptotic neutrophils contributes to the resolution of acute pulmonary inflammation in vivo. Am J Respir Cell Mol Biol. 1995; 12:232-237. [PubMed: 7865221]

Crews L, et al. Insights into the pathogenesis of hydrocephalus from transgenic and experimental animal models. Brain Pathol. 2004; 14:312-316. [PubMed: 15446587]

Ekinci N, et al. Volumetric evaluation of the relations among the cerebrum, cerebellum and brain stem in young subjects: a combination of stereology and magnetic resonance imaging. Surg Radiol Anat. 2008; 30:489-494. [PubMed: 18478176]

Fanaroff AA, et al. Trends in neonatal morbidity and mortality for very low birthweight infants. Am J Obstet Gynecol. 2007; 196:147 e1-147 e8. [PubMed: 17306659]

Faustino JV, et al. Microglial cells contribute to endogenous brain defenses after acute neonatal focal stroke. J Neurosci. 2011; 31:12992-13001. [PubMed: 21900578]

Fumagalli M, et al. From germinal matrix to cerebellar haemorrhage. J Matern Fetal Neonatal Med. 2015; 28(Suppl 1):2280-2285. [PubMed: 23968333]

Gonzales NR, et al. Design of a prospective, dose-escalation study evaluating the Safety of Pioglitazone for Hematoma Resolution in Intracerebral Hemorrhage (SHRINC). Int J Stroke. 2013; 8:388-396. [PubMed: 22340518]

Harry GJ, Kraft AD. Microglia in the developing brain: a potential target with lifetime effects. Neurotoxicology. 2012; 33:191-206. [PubMed: 22322212]

Hartman R, et al. Assessing functional outcomes following intracerebral hemorrhage in rats. Brain Res. 2009; 1280:148-157. [PubMed: 19464275]

Jain NJ, et al. Impact of mode of delivery on neonatal complications: trends between 1997 and 2005. J Matern Fetal Neonatal Med. 2009; 22:491-500. [PubMed: 19504405]

Kadri H, et al. The incidence, timing, and predisposing factors of germinal matrix and intraventricular hemorrhage (GMH/IVH) in preterm neonates. Childs Nerv Syst. 2006; 22:1086-1090. [PubMed: 16636880]

Keep RF, et al. The deleterious or beneficial effects of different agents in intracerebral hemorrhage: think big, think small, or is hematoma size important? Stroke. 2005; 36:1594-1596. [PubMed: 15933250]

Klebe D, et al. Acute and delayed deferoxamine treatment attenuates long-term sequelae after germinal matrix hemorrhage in neonatal rats. Stroke. 2014; 45:2475-2479. [PubMed: 24947291]

Klebe D, et al. Modulating the Immune Response Towards a Neuroregenerative Peri-injury Milieu After Cerebral Hemorrhage. J Neuroimmune Pharmacol. 2015

Kouadir M, et al. CD36 participates in $\operatorname{PrP}(106-126)$-induced activation of microglia. PLoS One. 2012; 7:e30756. [PubMed: 22292032]

Lackner P, et al. Chronic hydrocephalus after experimental subarachnoid hemorrhage. PLoS One. 2013; 8:e69571. [PubMed: 23936048]

Landreth G, et al. PPARgamma agonists as therapeutics for the treatment of Alzheimer's disease. Neurotherapeutics. 2008; 5:481-489. [PubMed: 18625459]

Lekic T, et al. Neonatal brain hemorrhage (NBH) of prematurity: translational mechanisms of the vascular-neural network. Curr Med Chem. 2015; 22:1214-1238. [PubMed: 25620100]

Lekic T, et al. Rodent neonatal germinal matrix hemorrhage mimics the human brain injury, neurological consequences, and post-hemorrhagic hydrocephalus. Exp Neurol. 2012; 236:69-78. [PubMed: 22524990]

Lekic T, et al. A novel preclinical model of germinal matrix hemorrhage using neonatal rats. Acta Neurochir Suppl. 2011; 111:55-60. [PubMed: 21725732]

MacLellan CL, et al. Intracerebral hemorrhage models in rat: comparing collagenase to blood infusion. J Cereb Blood Flow Metab. 2008; 28:516-525. [PubMed: 17726491]

Oorschot DE. Total number of neurons in the neostriatal, pallidal, subthalamic, and substantia nigral nuclei of the rat basal ganglia: a stereological study using the cavalieri and optical disector methods. J Comp Neurol. 1996; 366:580-599. [PubMed: 8833111]

Osterman MJ, et al. Annual Summary of Vital Statistics: 2012-2013. Pediatrics. 2015 
Penas F, et al. Treatment in vitro with PPARalpha and PPARgamma ligands drives M1-to-M2 polarization of macrophages from T. cruzi-infected mice. Biochim Biophys Acta. 2015; 1852:893904. [PubMed: 25557389]

Pereira MP, et al. The nonthiazolidinedione PPARgamma agonist L-796,449 is neuroprotective in experimental stroke. J Neuropathol Exp Neurol. 2005; 64:797-805. [PubMed: 16141790]

Pisanu A, et al. Dynamic changes in pro- and anti-inflammatory cytokines in microglia after PPARgamma agonist neuroprotective treatment in the MPTPp mouse model of progressive Parkinson's disease. Neurobiol Dis. 2014; 71:280-291. [PubMed: 25134730]

Reisert I, et al. The glial reaction in the course of axon regeneration: a stereological study of the rat hypoglossal nucleus. J Comp Neurol. 1984; 229:121-128. [PubMed: 6490973]

Ren Y, et al. CD36 gene transfer confers capacity for phagocytosis of cells undergoing apoptosis. J Exp Med. 1995; 181:1857-1862. [PubMed: 7536797]

Rios FJ, et al. Oxidized LDL induces alternative macrophage phenotype through activation of CD36 and PAFR. Mediators Inflamm. 2013; 2013:198193. [PubMed: 24062612]

Roberts D, Dalziel S. Antenatal corticosteroids for accelerating fetal lung maturation for women at risk of preterm birth. Cochrane Database Syst Rev. 2006:CD004454. [PubMed: 16856047]

Schaar KL, et al. Functional assessments in the rodent stroke model. Exp Transl Stroke Med. 2010; 2:13. [PubMed: 20642841]

Shankaran S, et al. Relationship between antenatal steroid administration and grades III and IV intracranial hemorrhage in low birth weight infants. The NICHD Neonatal Research Network. Am J Obstet Gynecol. 1995; 173:305-312. [PubMed: 7631710]

Shao ZQ, Liu ZJ. Neuroinflammation and neuronal autophagic death were suppressed via Rosiglitazone treatment: New evidence on neuroprotection in a rat model of global cerebral ischemia. J Neurol Sci. 2015; 349:65-71. [PubMed: 25623802]

Tang J, et al. Minocycline Attenuates Neonatal Germinal-Matrix-Hemorrhage-Induced Neuroinflammation and Brain Edema by Activating Cannabinoid Receptor 2. Mol Neurobiol. 2015

Tang J, et al. Mmp-9 deficiency enhances collagenase-induced intracerebral hemorrhage and brain injury in mutant mice. J Cereb Blood Flow Metab. 2004; 24:1133-1145. [PubMed: 15529013]

Tang Y, et al. Age-related change of the neuronal number in the human medial vestibular nucleus: a stereological investigation. J Vestib Res. 2001; 11:357-363. [PubMed: 12446961]

Vexler ZS, Yenari MA. Does inflammation after stroke affect the developing brain differently than adult brain? Dev Neurosci. 2009; 31:378-393. [PubMed: 19672067]

Volpe JJ. Cerebellum of the premature infant: rapidly developing, vulnerable, clinically important. J Child Neurol. 2009; 24:1085-1104. [PubMed: 19745085]

Wang J, Dore S. Heme oxygenase-1 exacerbates early brain injury after intracerebral haemorrhage. Brain. 2007; 130:1643-1652. [PubMed: 17525142]

Woo MS, et al. Genetic deletion of CD36 enhances injury after acute neonatal stroke. Ann Neurol. 2012; 72:961-970. [PubMed: 23280844]

Xi G, et al. Mechanisms of brain injury after intracerebral haemorrhage. Lancet Neurol. 2006; 5:5363. [PubMed: 16361023]

Yoon HJ, et al. Regulation of TLR2 expression by prostaglandins in brain glia. J Immunol. 2008; 180:8400-8409. [PubMed: 18523308]

Zhao X, et al. Hematoma resolution as a therapeutic target: the role of microglia/macrophages. Stroke. 2009; 40:S92-S94. [PubMed: 19064796]

Zhao X, et al. Cleaning up after ICH: the role of Nrf2 in modulating microglia function and hematoma clearance. J Neurochem. 2015; 133:144-152. [PubMed: 25328080]

Zhao X, et al. Hematoma resolution as a target for intracerebral hemorrhage treatment: role for peroxisome proliferator-activated receptor gamma in microglia/macrophages. Ann Neurol. 2007; 61:352-362. [PubMed: 17457822] 


\section{Highlights}

- Treatment with $15 \mathrm{~d}-\mathrm{PGJ}_{2}$ after germinal matrix hemorrhage in neonatal rats

- PPAR $\gamma$ stimulation, CD36 scavenger receptor, and microglia/macrophage activation

- Enhanced hematoma resolution and improved long-term brain morphological outcomes

- Attenuated long-term neurofunctional deficits 


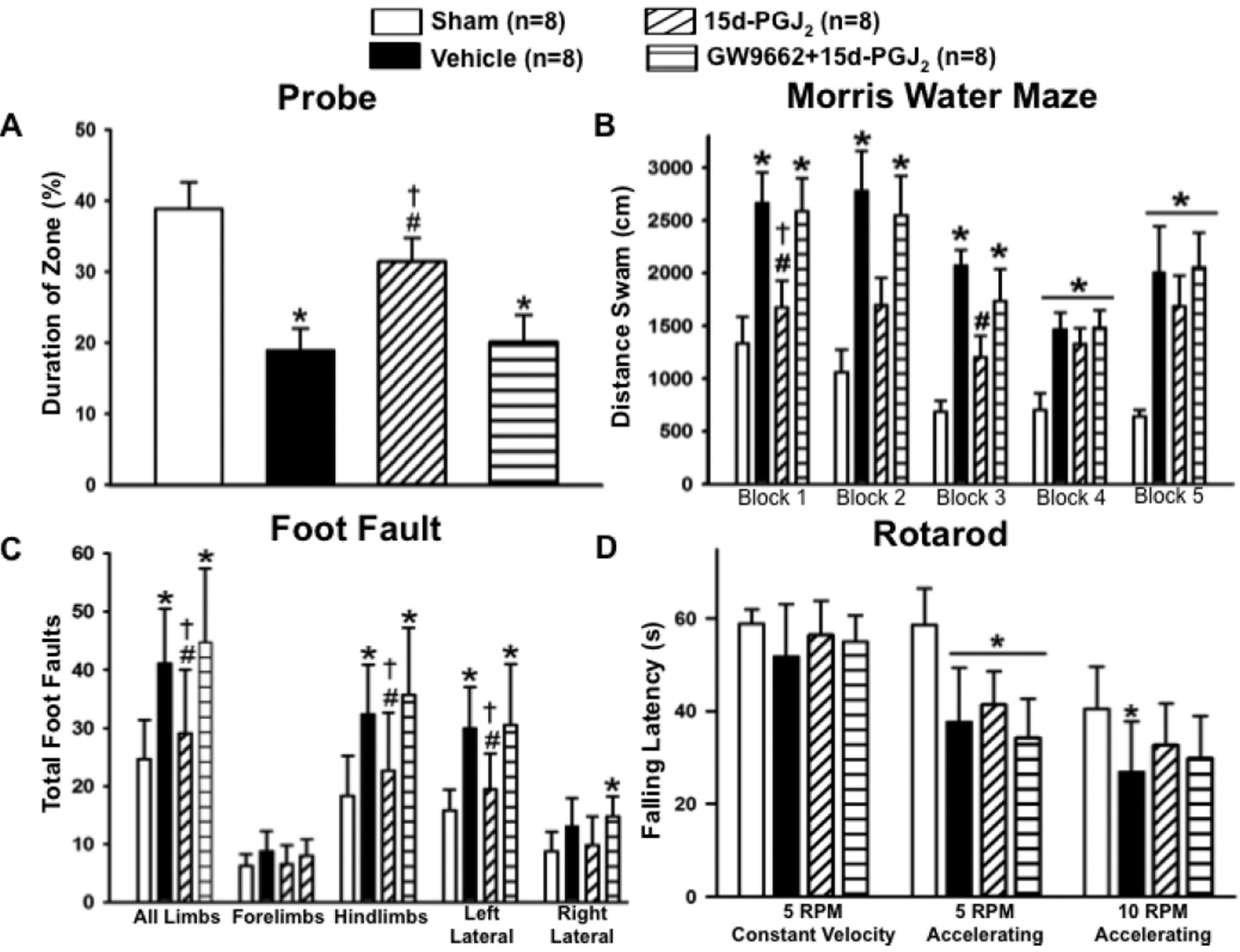

Figure 1.

Neurofunctional assessment of (A and B) Morris water maze, (C) foot fault, and (D) rotarod at 21 to 28 days after germinal matrix hemorrhage. Values are expressed as mean $\pm \mathrm{SD}$.

$* \mathrm{P}<0.05$ compared with sham, $\# \mathrm{P}<0.05$ compared with vehicle, and $\dagger \mathrm{P}<0.05$ compared with inhibitor and agonist. $\mathrm{N}=8$ per group; and RPM, rounds per minute. 
A

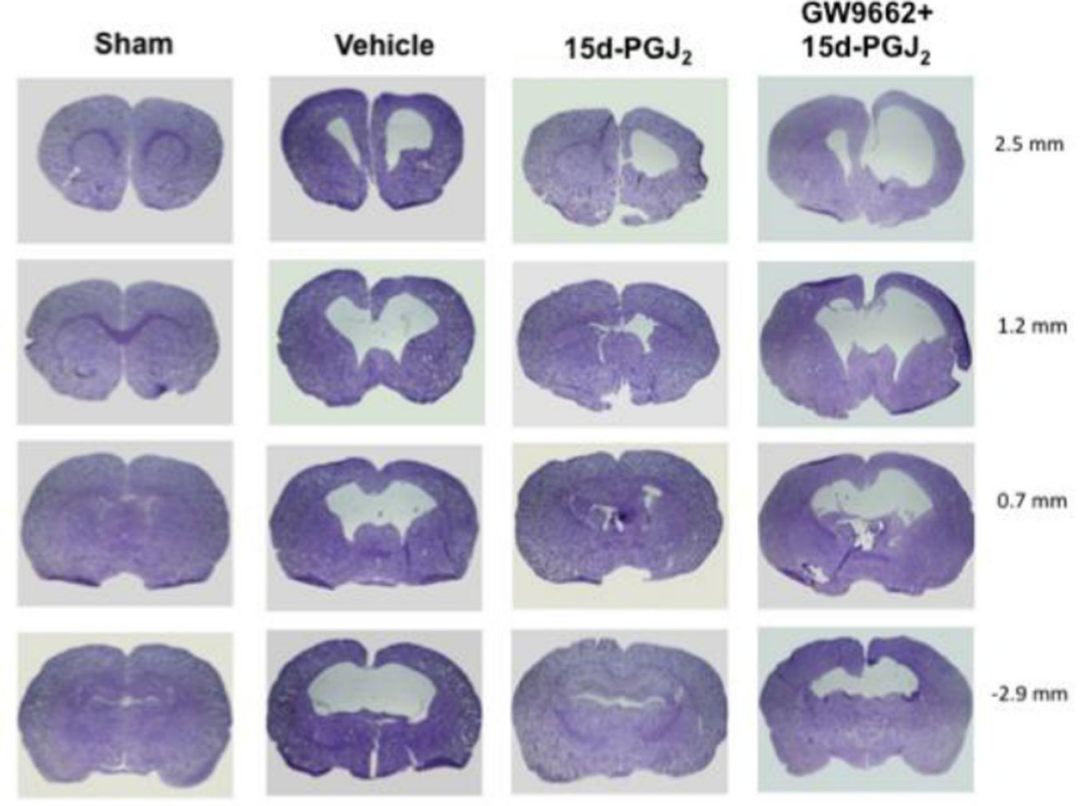

B

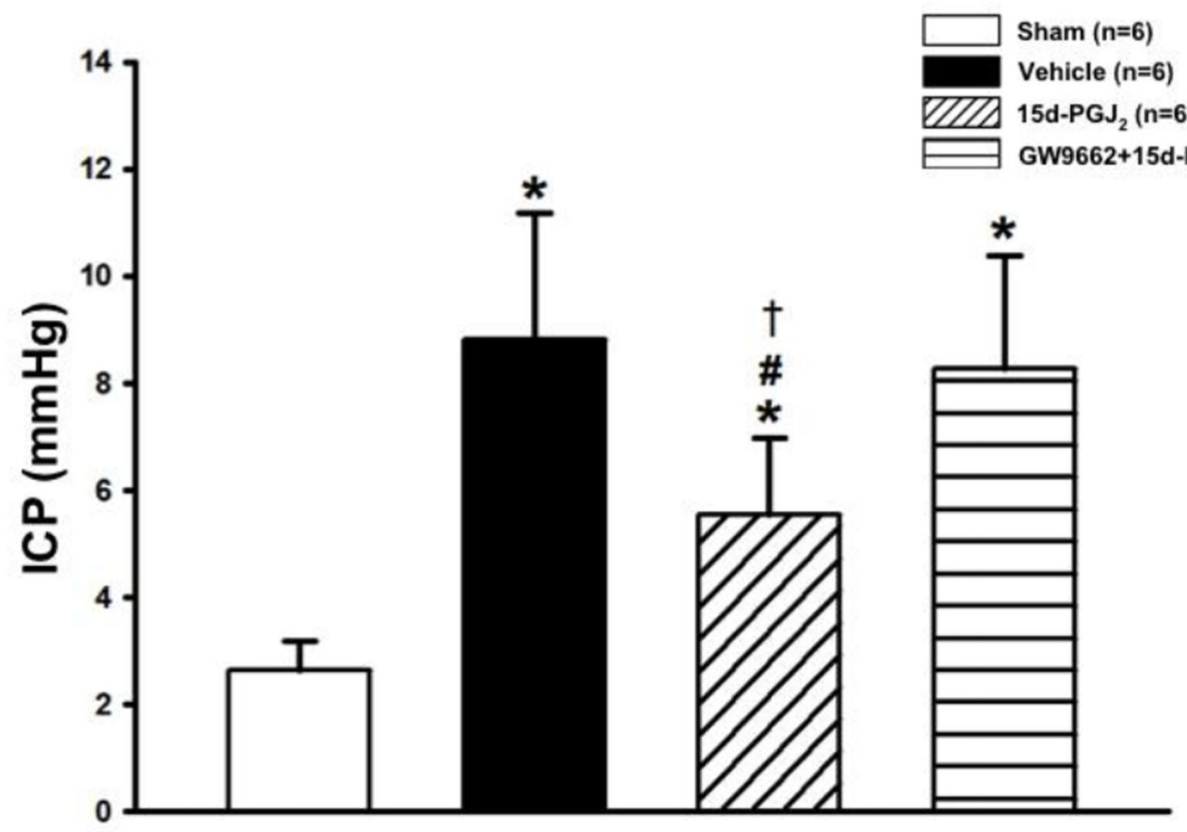

Figure 2.

28 days after germinal matrix hemorrhage. Representative microphotographs of (A) Nisslstained brain sections were taken and (B) Intracranial pressure (ICP) in mmHg. Note: measurement on the right side of the representative pictures indicats the location of the brain section from bregma. Values are expressed as mean $\pm \mathrm{SD}$. $* \mathrm{P}<0.05$ compared with sham, $\# \mathrm{P}<0.05$ compared with vehicle, and $\dagger \mathrm{P}<0.05$ compared with inhibitor and agonist. $\mathrm{N}=6$ per group; and mmHG, millimeters of Mercury. 


$\begin{array}{ll}\square \text { Sham }(n=6) & \square 15 d-P G J_{2}(n=6) \\ \text { Vehicle }(n=6) & \text { GW9662+15d-PGJ }(n=6)\end{array}$

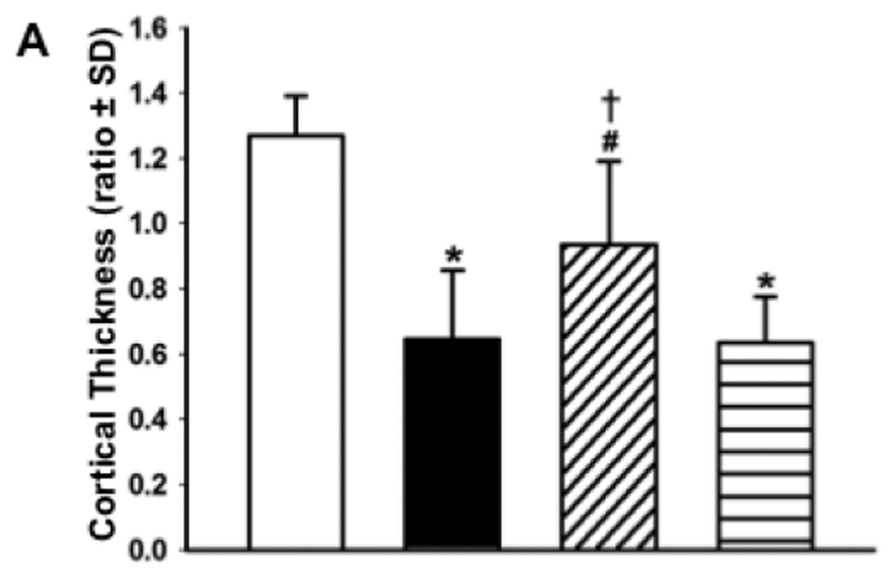

B

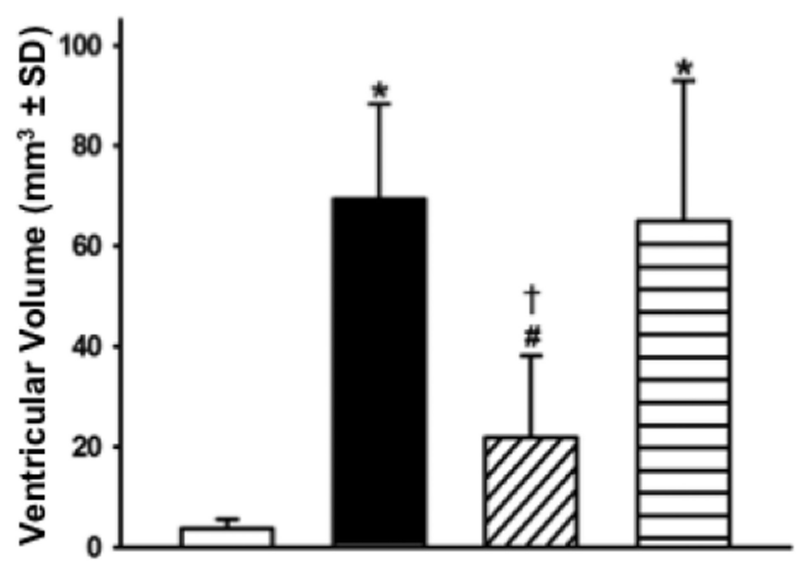

C
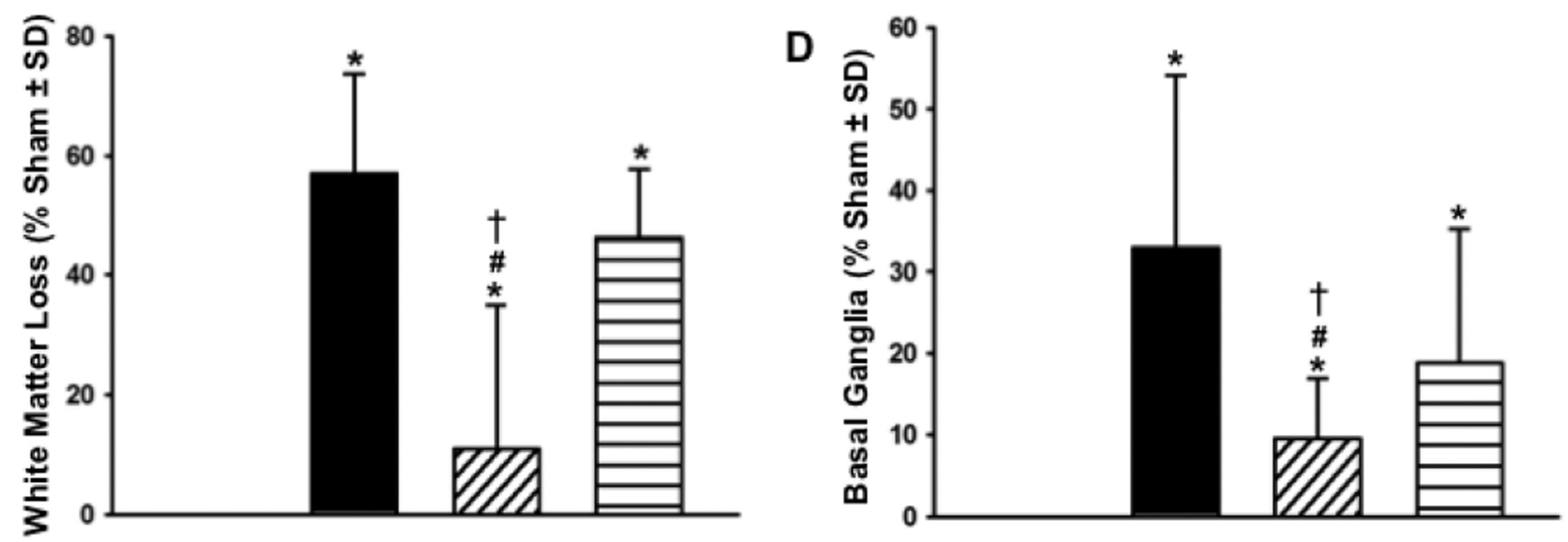

Figure 3.

Quantification of (A) cortical thickness, $(\mathbf{B})$ ventricular volume, $(\mathbf{C})$ white matter loss, and (D) basal ganglia loss at 28 days after germinal matrix hemorrhage. Values are expressed as mean \pm SD. $* \mathrm{P}<0.05$ compared with sham, $\# \mathrm{P}<0.05$ compared with vehicle, and $\uparrow \mathrm{P}<0.05$ compared with inhibitor and agonist. $\mathrm{N}=6$ per group. 
A

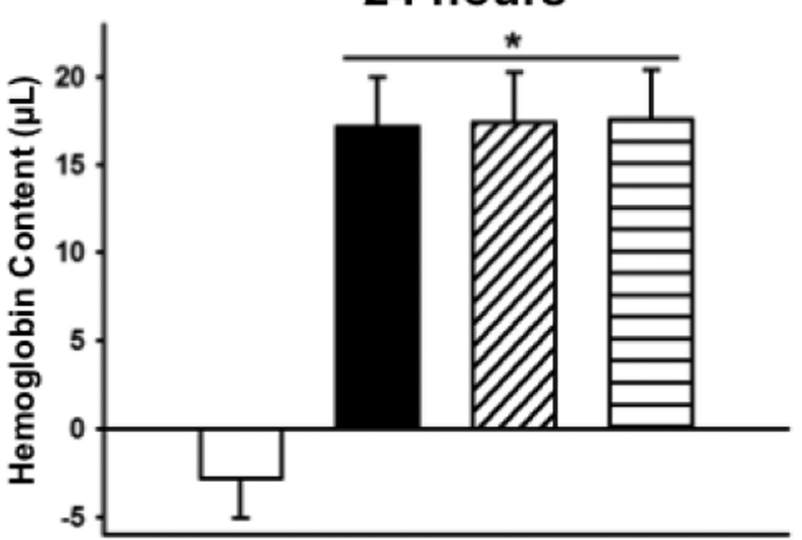

C

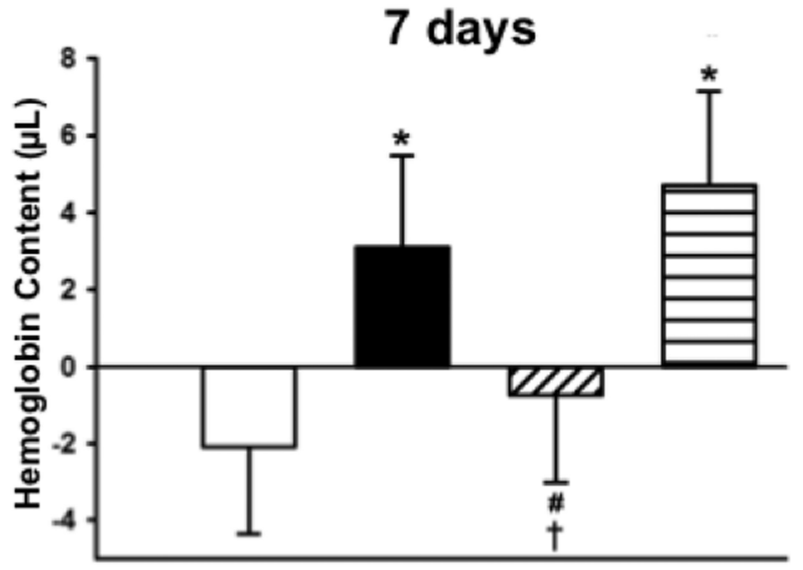

B

\section{2 hours}

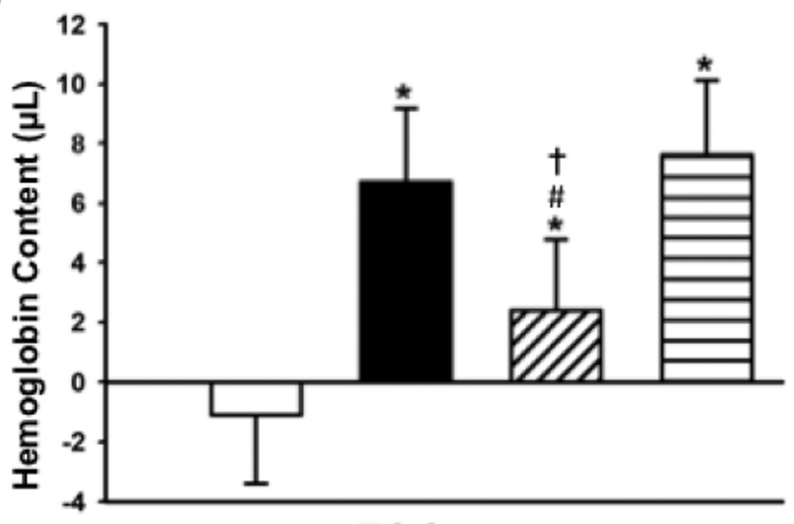

72 hours

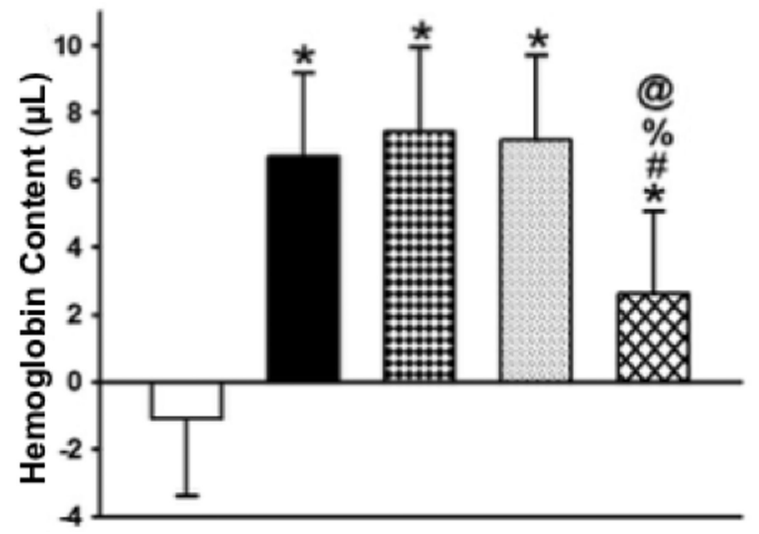

Figure 4.

Hemoglobin assay at (A) 24 hours, (B) 72 hours, and (C) 7 days. At 72 hours (D)

Hemoglobin assay was conducted on siRNA groups. Values are expressed as mean \pm SD.

$* \mathrm{P}<0.05$ compared with sham, $\# \mathrm{P}<0.05$ compared with vehicle, and $\dagger \mathrm{P}<0.05$ compared with inhibitor and agonist, \% $\mathrm{P}<0.05$ compared CD 36 siRNA, and @ $\mathrm{P}<0.05$ compared to scrambled siRNA. $\mathrm{N}=6$ per group. 
A

ㅁ
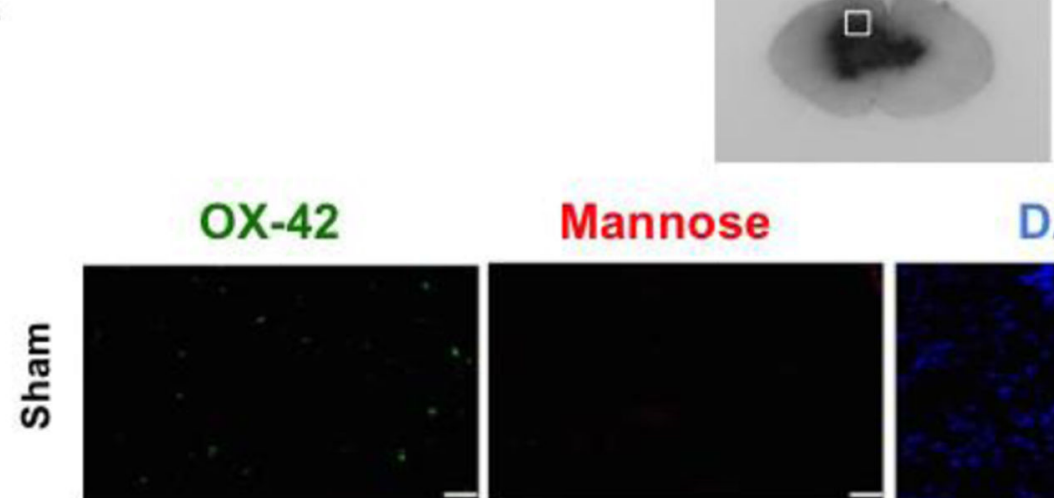

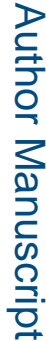

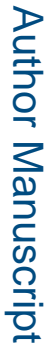
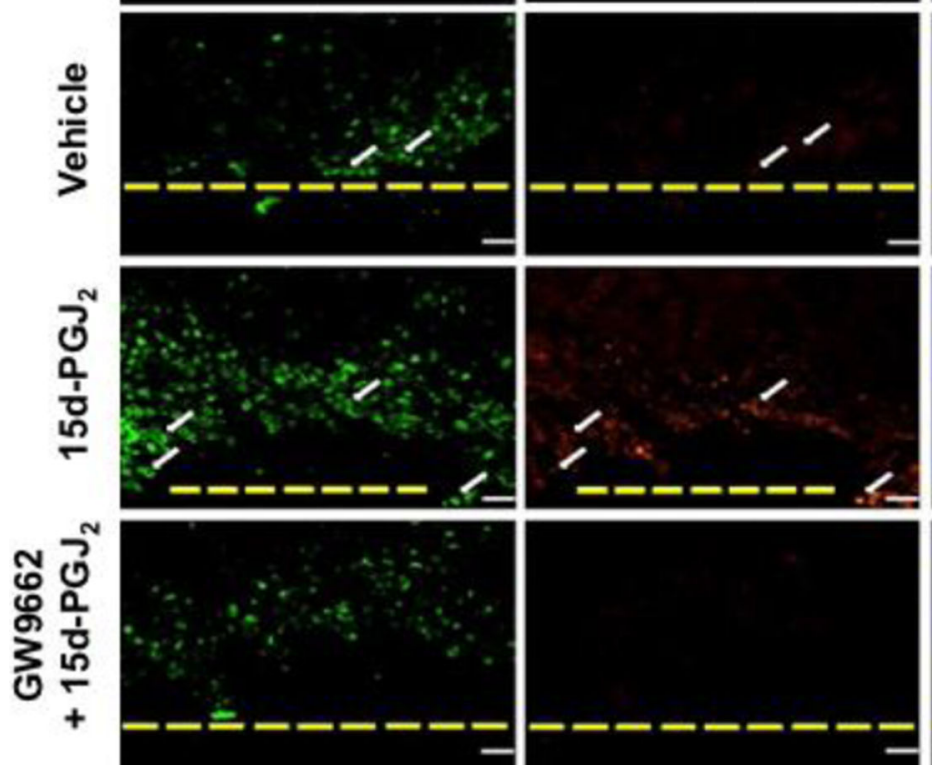

DAPI

OX-42/ Mannose/ DAPI
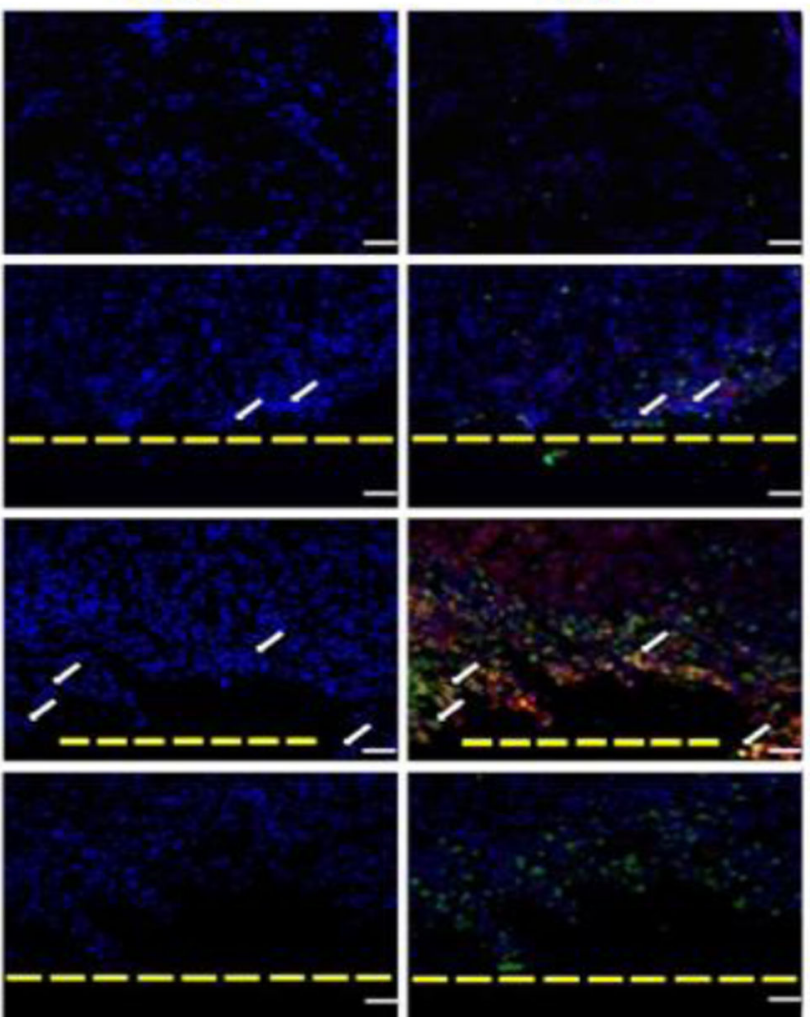

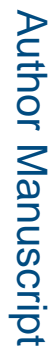


B

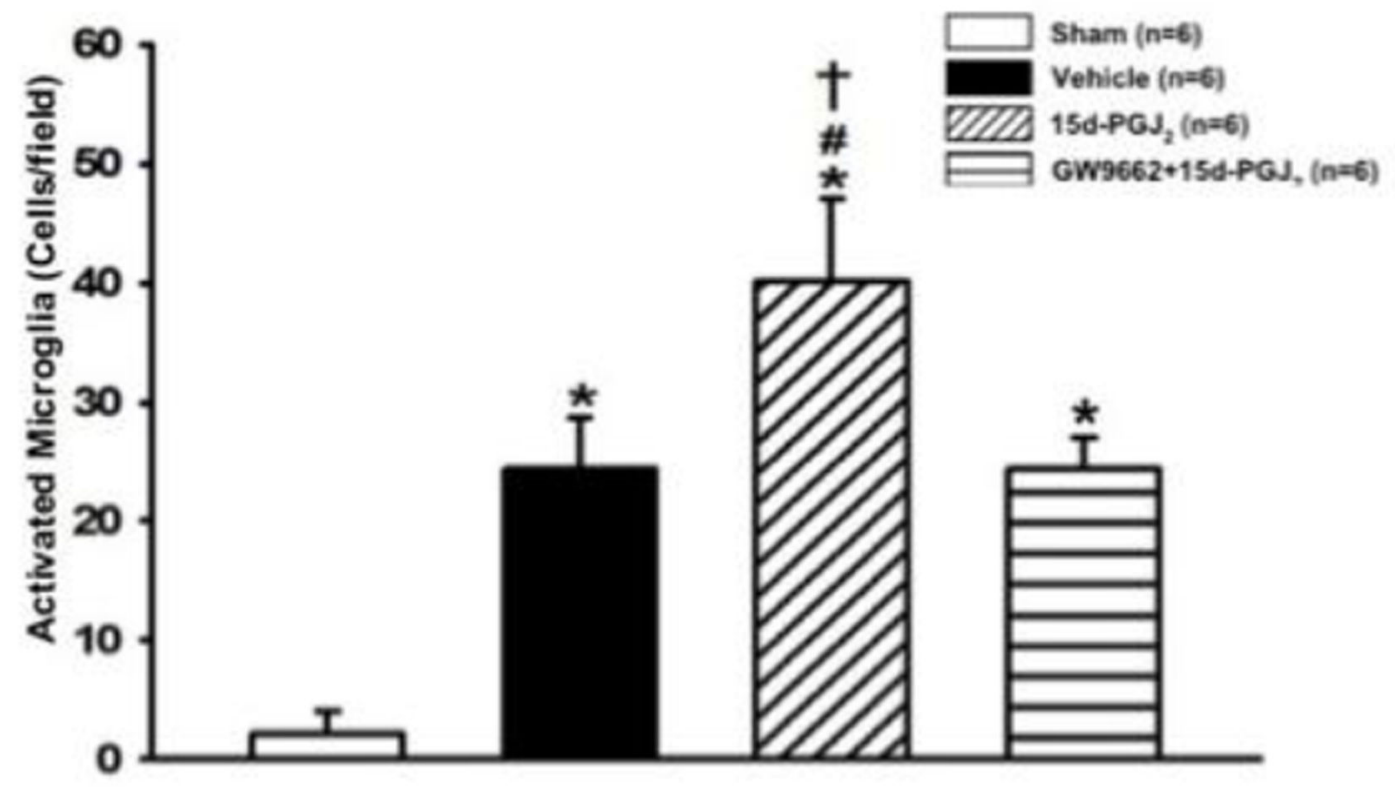

C

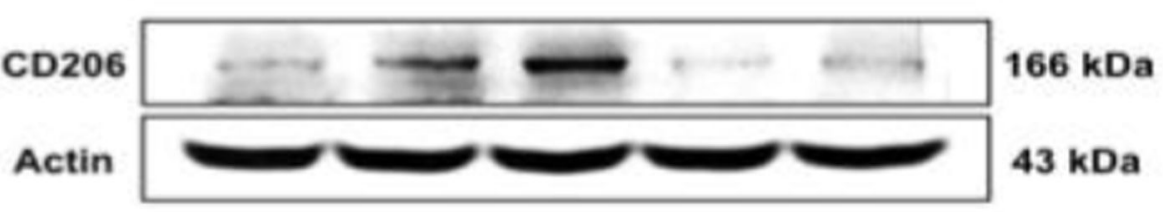

\section{CD206 Expression at 72 Hours Post-ictus}

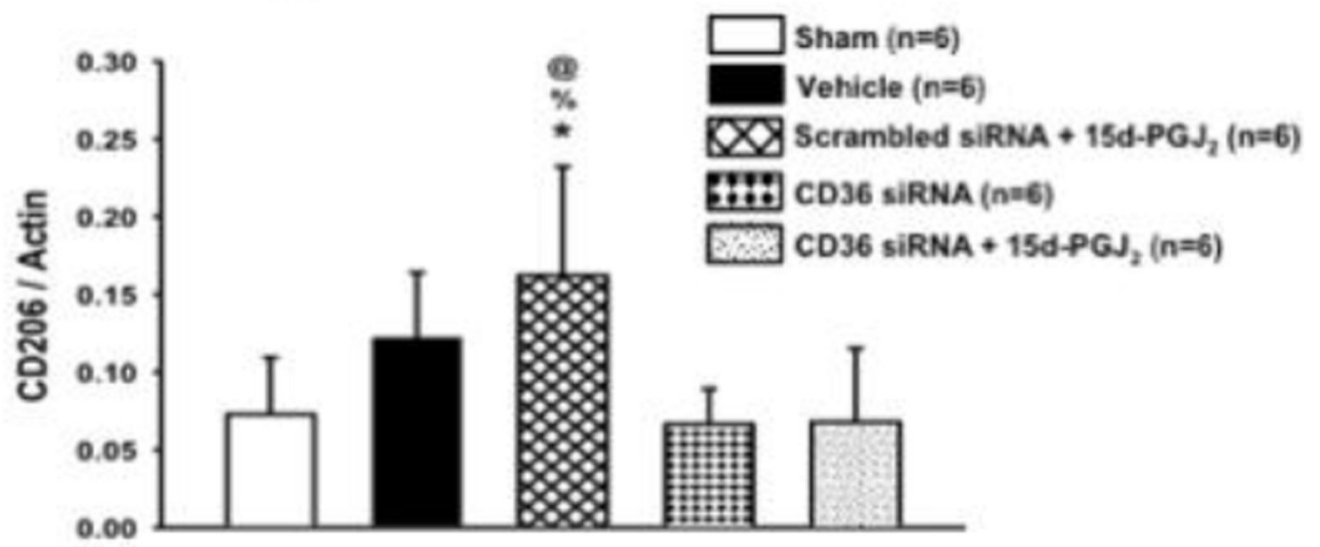

Figure 5.

Immunohistochemistry representative pictures were taken showing the co-localization of (A) activated microglia (OX-42; scale bar: $20 \mu \mathrm{m}$ ) with Mannose Receptor and DAPI staining and (B) quantification of activated microglia was conducted at 72 hours. Western blots were conducted at 72 hours for (C) CD206 Expression. Note: peri-hematoma region is below the yellow broken line (A) and the representative GMH brain indicates where IHC images were taken. Values are expressed as mean \pm SD. $* \mathrm{P}<0.05$ compared with sham, \#P<0.05 compared with vehicle, $\uparrow \mathrm{P}<0.05$ compared with inhibitor and agonist, $\% \mathrm{P}<0.05$ compared CD 36 
siRNA, and @ $\mathrm{P}<0.05$ compared to scrambled siRNA. N=6 per group; and $\mathrm{ICH}$, Immunohistochemistry. $\mathrm{N}=6$ per group. 
A PPARy

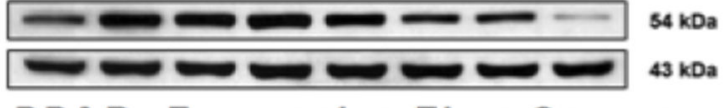

PPAR $\gamma$ Expression Time Course

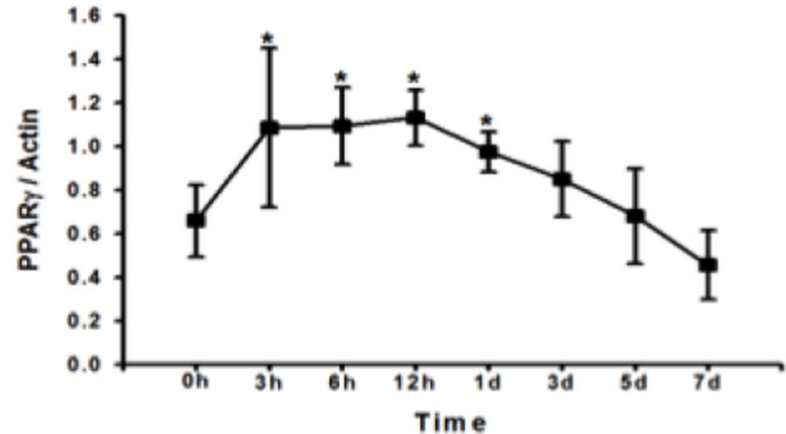

C
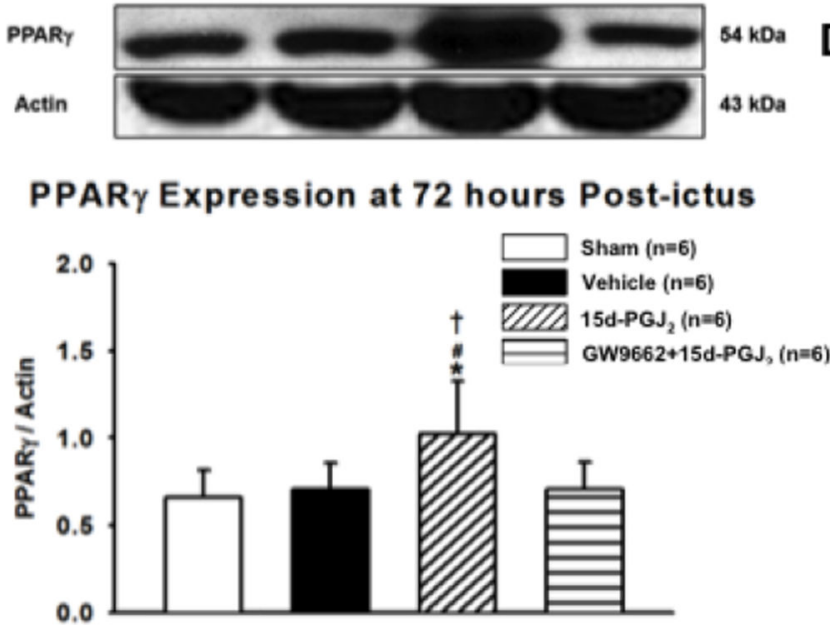

Figure 6.
B ${ }_{\text {Actin }}^{\mathrm{CD} 36}$

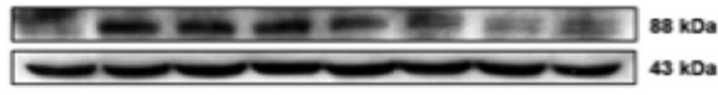

CD 36 Expression Time Course
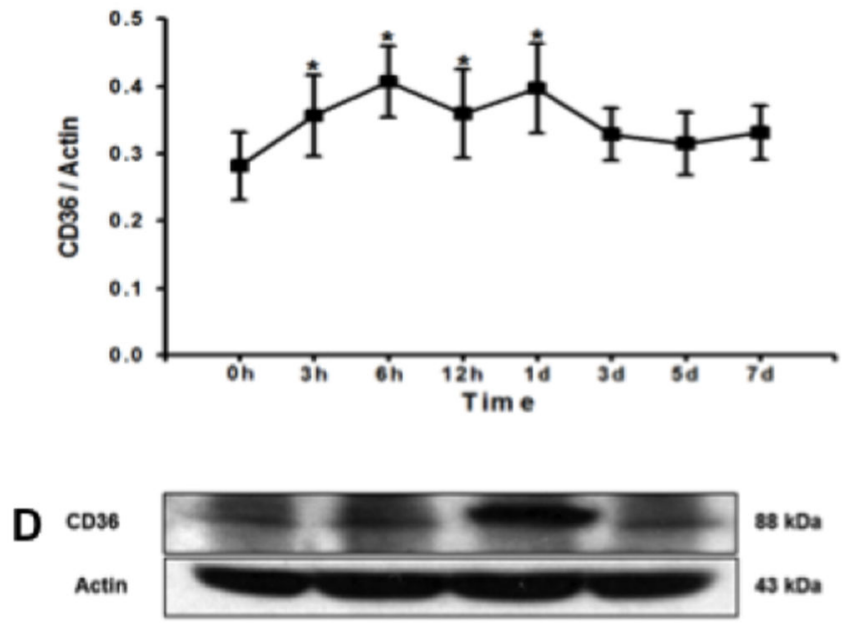

CD36 Expression at 72 hours Post-ictus

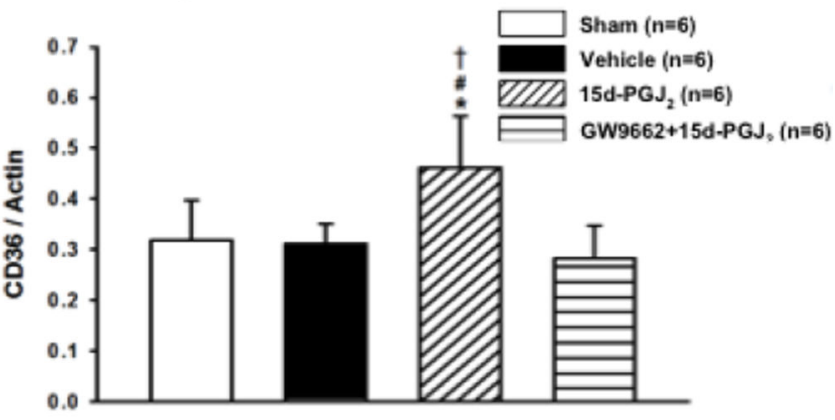

Western blot was conducted for time-course studies of (A) PPAR $\Upsilon$ and (B) CD36 at 0, 3, 6, 12 hours, 1, 3, 5, 7 days after GMH. Western blot was then conducted at 72 hours for $(\mathbf{C})$ PPAR- $\Upsilon$ and (D) CD36. Values are expressed as mean \pm SD. $* \mathrm{P}<0.05$ compared with sham, $\# \mathrm{P}<0.05$ compared with vehicle, and $\dagger \mathrm{P}<0.05$ compared with inhibitor and agonist. $\mathrm{N}=6$ per group; and $\mathrm{h}$ and $\mathrm{d}$, hour(s) and day(s). 